\title{
Gram positive bugs - bugging us in today's era. A study from a tertiary care hospital in Punjab (North India)
}

\author{
Eshani Dewan*, Aroma Oberoi \\ Department of Microbiology, Christian Medical College and Hospital, Ludhiana \\ *Corresponding author E-mail: eshanidewan@yahoo.com
}

Copyright $\odot 2014$ Eshani Dewan and Aroma Oberoi. This is an open access article distributed under the Creative Commons Attribution License, which permits unrestricted use, distribution, and reproduction in any medium, provided the original work is properly cited.

\begin{abstract}
Background: Recently there has been a rapid increase in the incidence of infection and colonization of patients with gram positive organisms such as Enterococcus and Staphylococcus which display resistance to many commonly used antimicrobial agents.

Materials and methods: A prospective study was conducted over a period of four months in the department of Microbiology of a North Indian hospital. Various clinical samples were collected from the patients, the isolates were identified using standard microbiological methods and subjected to in vitro antimicrobial susceptibility testing by disc diffusion method and newer antimicrobials such as Vancomycin, Teicoplanin and Daptomycin by E test also.

Results: A total of 220 isolates of, Enterococci (132) and MRSA (88) were tested. Resistance among Enterococci to various drugs was; Penicillin (88.63\%), Erythromycin (76.06\%), high level Gentamycin (51.03\%) and Ciprofloxacin (46.21\%). By disc diffusion method Vancomycin and Teicoplanin showed $15.15 \%$ and $7.58 \%$ resistance. Whereas, E test demonstrated lower resistance to Vancomycin (11.36\%) and Teicoplanin (9.09\%). Resistance among MRSA to Erythromycin, Ciprofloxacin, Clindamycin, Vancomycin and Teicoplanin was 64.77, 48.87, 62.50, 4.54 and 2.27 per cent, respectively. Zero percent resistance was seen towards Linezolid. By E test resistance to Daptomycin was $2.27 \%$, whereas all the isolates were sensitive to Vancomycin and Teicoplanin.

Conclusions: The spread of antibiotic resistance among organisms, to the point where some isolates are resistant to all standard therapies highlights the looming prospect of a 'post antibiotic era'. Antibiotic policies need to be updated regularly, along with comprehensive monitoring of antibiotic prescription and consumption.
\end{abstract}

Keywords: Gram Positive Organisms, E Test, Vancomycin, Teicoplanin and Daptomycin.

\section{Introduction}

Enterococci and Staphylococci have evolved over the past century from being commensal organisms of little clinical significance to becoming one the most common pathogens associated with significant morbidity and mortality. In recent years, there has been a rapid increase in the incidence of infection and colonisation of patients with these gram positive organisms. The resistance may be intrinsic or acquired via gene transfer. This is mainly attributable to widespread use of extended-spectrum cephalosporins and Vancomycin in hospitals which has likely contributed to the emergence and dramatic increase of resistant organisms over the past 20 years [1]. Their emergence in past two decades is in many respects due to their resistance to many commonly used antimicrobial agents (aminoglycosides, cephalosporins, Aztreonam, semisynthetic Penicillin, Trimethoprim-Sulphamethoxazole) and ease with which they appear to attain and transfer resistant genes, thus giving rise to high level aminoglycoside resistance (HLAR), $\beta$-lactamase production and glycopeptide resistance [2]. S. aureus strains resistant to Vancomycin have been obtained in vitro either by selection of resistant mutants or by the conjugal transfer of genes encoding Vancomycin resistance from Enterococci. Teicoplaninresistant derivatives of Teicoplanin-susceptible $S$. aureus strains have also been obtained in vitro [3]. Vancomycin Resistant Enterococcus (VRE) is another organism of great concern since it was first reported in France in 1988. Since then, the incidence of VRE has been reported in many parts of the world. The number of cases infected with VRE, as reported by the US Centre for Disease Control and Prevention, has risen from 99 in 1992 to 278 in 1994 . The more 
recent reports are both distressing as well as alarming [4]. Therefore, the present study was undertaken to check susceptibility of MRSA and Enterococcus isolates against new antimicrobials such as Vancomycin, Teicoplanin, Linezolid and Daptomycin via the disc diffusion method and MIC by E test in Christian Medical College, Ludhiana.

\section{Material and methods}

This study was conducted over a period of 4 months in Christian Medical College, Ludhiana, where a total of 220 isolates were evaluated from various clinical samples such as blood, pus, urine etc. Out of which 132 were Enterococci and 88 MRSA. The distribution of MRSA isolates was; pus $(n=75)$, urine $(n=10)$, body fluid and blood $(n=3)$. Whereas, the Enterococci isolates that were obtained were from; urine $(n=65)$, pus $(n=54)$ and blood $(n=13)$. All Staphylococcal and Enterococcal isolates were identified by standard microbiological methods [5], [6].

Antimicrobial susceptibility testing

The susceptibilities of all isolates to different antimicrobial agents were tested by Kirby-Bauer disc-diffusion method as standardised by the Clinical Laboratory Standards Institution (CLSI) [7]. The following antimicrobials were used for Enterococci: Penicillin $(10 \mu \mathrm{g})$, Ampicillin $(10 \mu \mathrm{g})$, Erythromycin $(15 \mu \mathrm{g})$, Ciprofloxacin $(5 \mu \mathrm{g})$, high level Gentamicin $(120 \mu \mathrm{g})$, Vancomycin $(30 \mu \mathrm{g})$, Teicoplanin $(30 \mu \mathrm{g})$ and Linezolid $(30 \mu \mathrm{g})$. For MRSA: Erythromycin $(15 \mu \mathrm{g})$, Ciprofloxacin $(5 \mu \mathrm{g})$, Cotrimoxazole $(1.25 / 23.75 \mu \mathrm{g})$, Clindamycin $(2 \mu \mathrm{g})$, Cefoxitin $(30 \mu \mathrm{g})$, Vancomycin $(30 \mu \mathrm{g})$, Teicoplanin $(30 \mu \mathrm{g})$ and Linezolid $(30 \mu \mathrm{g})$ were tested. The inhibition zone diameters were measured to the nearest millimetre and recorded. Each bacterial isolate was classified as susceptible (S), intermediate (I) and resistant (R) to antibiotic according to the zone diameter interpretation standard recommended by the Clinical Laboratory Standards Institute (CLSI). E. faecalis ATCC 29212 and S. aureus ATCC 25923 were used as quality control strains to check antibiotic discs and accuracy of the testing procedure. MRSA isolates were identified using Cefoxitin $(30 \mu \mathrm{g})$ disc diffusion method as per CLSI 2013[8].

Minimum inhibitory concentrations (MICs) for Vancomycin, Teicoplanin and Daptomycin were determined using Etest strips (bio Me'rieux). The table below shows the various MIC values in $\mu \mathrm{g} / \mathrm{ml}$ which were read as per the manufacturer's recommendation and interpretation made as per CLSI criteria [8]. All Staphylococcal isolates with MIC between 4 - $8 \mu \mathrm{g} / \mathrm{ml}$ were called Vancomycin Intermediate Susceptible Staph aureus (VISA) strains.

Table 1: MIC for Vancomycin, Teicoplanin and Daptomycin as Per CLSI Criteria

\begin{tabular}{|c|c|c|c|c|c|c|c|c|c|}
\hline & \multicolumn{3}{|c|}{ Vancomycin } & \multicolumn{3}{|c|}{ Teicoplanin } & \multicolumn{3}{|c|}{ Daptomycin } \\
\hline & $\mathrm{S}$ & I & $\mathrm{R}$ & $\mathrm{S}$ & $\mathrm{I}$ & $\mathrm{R}$ & $\mathrm{S}$ & $\mathrm{I}$ & $\mathrm{R}$ \\
\hline Enterococcus & $\leq 4$ & 8- 16 & $\geq 32$ & $\leq 8$ & 16 & $\geq 32$ & $\leq 4$ & - & - \\
\hline Staphylococcus & $\leq 2$ & $4-8$ & $\geq 16$ & $\leq 8$ & 16 & $\geq 16$ & $\leq 1$ & - & - \\
\hline
\end{tabular}

\section{Results}

A total of 220 Enterococci and Methicillin resistance Staphylococci isolates were obtained from different clinical samples from various wards/ ICUs/OPD. Among gram positive organisms Enterococcus accounted for [132(60\%)] and MRSA [88(40\%)] of the total isolates. The majority of Enterococci were obtained from urine [65(49.24\%)], followed by pus/wound swabs [54 (40.90\%)] and blood [13 (9.86\%)].

MRSA was isolated most frequently from pus/wound swabs [75(85.22)], followed by urine [10(11.36\%)] and blood $[3(3.42 \%)]$.

Table 2: Sample wise prevalence of isolates of Enterococcus and MRSA

\begin{tabular}{lll}
\hline Sample & Enterococcus $(\%)$ & MRSA (\%) \\
\hline Pus/wound swabs & $54(40.90)$ & $75(85.22)$ \\
Urine & $65(49.24)$ & $10(11.36)$ \\
Blood & $13(9.86)$ & $3(3.42)$ \\
Total & 132 & 88 \\
\hline
\end{tabular}

Figures in parenthesis show the percentage distribution 


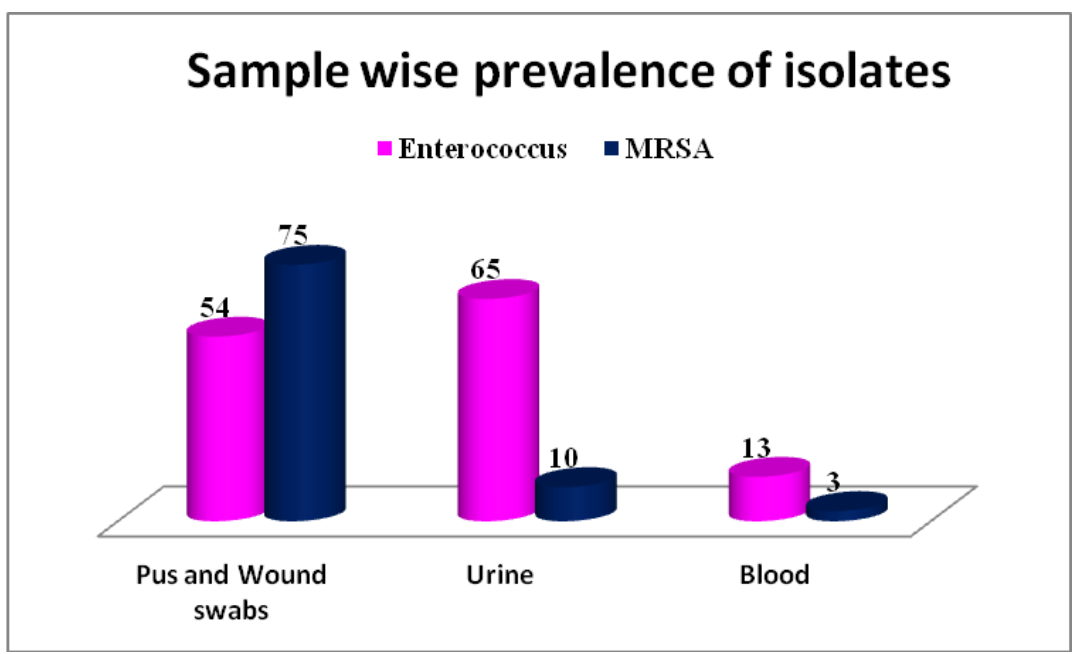

Fig. 1: Sample Wise Prevalence of Isolates of Enterococcus and MRSA

Resistance among 132 clinical isolates of Enterococci to drugs such as Penicillin was 88.63 per cent, Erythromycin 76.06 per cent; Ampicillin 31.81 per cent, high level Gentamycin was 51.03 per cent and Ciprofloxacin 46.21 per cent. By disc diffusion method, Enterococcus showed 15.15 per cent and 7.58 percent resistance to Vancomycin and Teicoplanin respectively. Whereas, E test demonstrated a lower resistance of 11.36 per cent to Vancomycin and 9.09 per cent to Teicoplanin. Three out of the 132 isolates showed resistance to Daptomycin.

Resistance among MRSA to Erythromycin, Ciprofloxacin, Cotrimazole, Clindamycin, Vancomycin and Teicoplanin was $64.77,48.87,52.75,62.50,4.54$ and 2.27 per cent, respectively. Zero percent resistance was found to Linezolid among both Enterococcal and MRSA isolates. All MRSA isolates were sensitive to Vancomycin and Teicoplanin by E test. Daptomycin was resistant in 2.27 per cent of MRSA isolates.

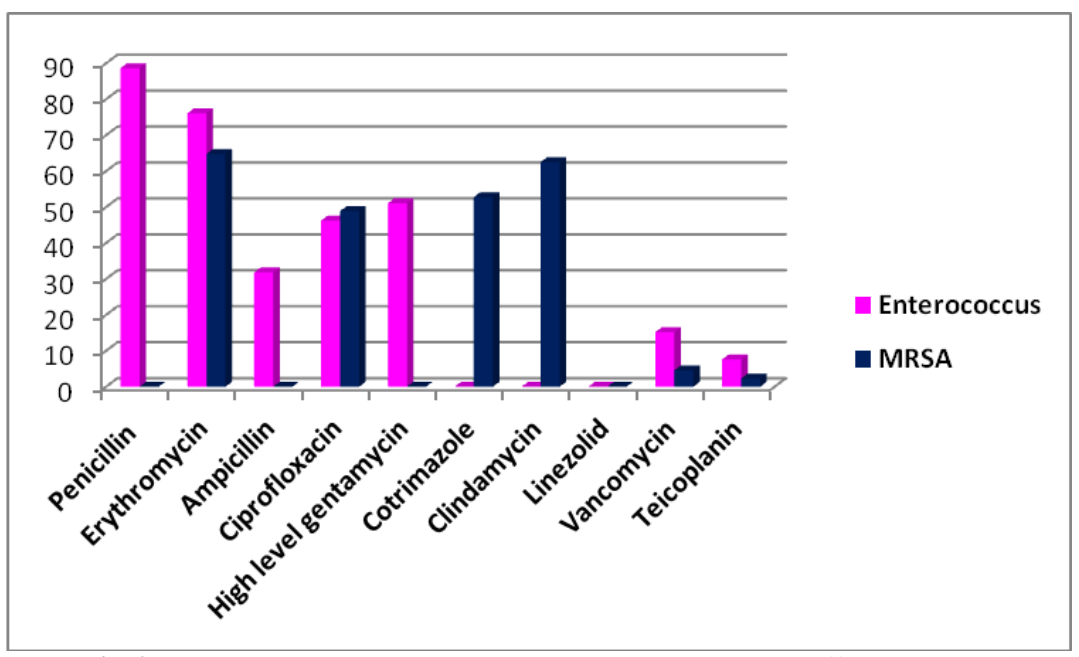

Fig. 2: Resistance among Enterococcus and MRSA as Per Disc Diffusion Method

Table 3: Percentage of resistance among Enterococcus and MRSA among various antibiotics

\begin{tabular}{|c|c|c|}
\hline \multirow{2}{*}{ Antibiotic } & \multicolumn{2}{|c|}{ \% Isolates Resistant (by disc diffusion) } \\
\hline & Enterococcus $(n=132)$ & MRSA $(n=88)$ \\
\hline Penicillin & 88.63 & - \\
\hline Erythromycin & 76.06 & 64.77 \\
\hline Ampicillin & 31.81 & - \\
\hline Ciprofloxacin & 46.21 & 48.87 \\
\hline High level Gentamycin & 51.03 & - \\
\hline Cotrimazole & - & 52.75 \\
\hline Clindamycin & - & 62.50 \\
\hline Linezolid & 0 & 0 \\
\hline Vancomycin & 15.15 & 4.54 \\
\hline Teicoplanin & 7.58 & 2.27 \\
\hline
\end{tabular}


Table 4: MIC by E test for Enterococcal and MRSA isolates

\begin{tabular}{lccccccccc}
\hline & \multicolumn{3}{c}{ Vancomycin } & \multicolumn{3}{c}{ Teicoplanin } & \multicolumn{3}{c}{ Daptomycin } \\
\hline & $\mathrm{S}$ & $\mathrm{I}$ & $\mathrm{R}$ & $\mathrm{S}$ & $\mathrm{I}$ & $\mathrm{R}$ & $\mathrm{S}$ & $\mathrm{I}$ & $\mathrm{R}$ \\
Enterococcus & $110(83.34)$ & $7(5.3)$ & $15(11.36)$ & $115(87.21)$ & $5(3.70)$ & $12(9.09)$ & $129(97.73)$ & - & $3(2.27)$ \\
Staphylococcus & $83(94.32)$ & $5(5.68)$ & $0(0)$ & $84(94.45)$ & $4(4.55)$ & $0(0)$ & $86(97.73)$ & - & $2(2.27)$ \\
\hline
\end{tabular}

Figures in parenthesis show the percentage distribution

\section{Discussion}

S. aureus and Enterococcus have been one of the most common pathogens in the past causing significant pyogenic, local and systemic infections in both hospitals and community. By virtue of a battery of virulence factors they have propensity to cause a wide and varied spectrum of infections which are frequently fatal in nature, such as, meningitis, endocarditis and blood stream infections [1]. Progress in medical technology and injudicious intensive use of broad spectrum antibiotics in the hospitals has been responsible for emergence of new strains of these organisms and replacement of the previous strains. These multi-drug resistant strains have now spread in the community to cause community acquired infections [9].

Enterococci has emerged as a major cause of nosocomial infections, recognized as the 3rd most common cause of bacteraemia and the second most common cause of hospital acquired urinary tract infections (second only to E.coli) [10], [11]. In our study it accounted for $60 \%$ of the total isolates, among which Vancomycin resistant Enterococcus (VRE) accounted for $11.36 \%$.VRE is known to cause significant mortality and morbidity [4]. There are very few reports on isolation of VRE from India [2]. Similar rates of VRE were reported by a number of studies that have been conducted in India [12], [13], [14] although; a study in Navi Mumbai showed much higher percentage (26\%) [15]. VRE can remain viable in the environment for an extended time period, and therefore poise a problem for infection control in hospitals and nursing homes. In addition, these Enterococci have been detected as part of the enteric flora in nonsymptomatic patients. These colonized patients serve as potential sources for transfer of this organism to other patients and medical personnel. Studies propose that prolonged hospital stay and the irrational use of Vancomycin are important risk factors for Vancomycin resistance in Enterococci. Other significant factors that were associated with the emergence of VRE were renal dialysis and or renal failure, prior amino glycoside use and the use of third generation cephalosporins. Importance of screening for VRE in clinical samples is also being emphasized. In practice it is not always easy to assess the clinical significance of VRE in routine culture thus it is important to differentiate colonisation from infection [4]. The Enterococcus isolates with high MIC for Vancomycin also had high MIC for Teicoplanin suggesting cross-resistance between the two drugs [12]. The increased MIC for Vancomycin in S. aureus and Enterococci could possibly be due to increased usage of the drug at our centre.

Methicillin Resistant $S$. aureus (MRSA) are strains of $S$. aureus which express altered penicillin binding protein (PBP2a), thus conferring resistance to beta lactam antibiotics. MRSA strains had caused several documented outbreaks of hospital cross infections throughout the world in 1970s and since then, they have drawn special attention in hospital acquired infections. While MRSA is less in countries like Norway and Sweden (1\%), Canada (5-10\%), it is 25-50\% in the United States and 43\%-58\% in Italy. High prevalence of MRSA is an emerging problem in India. In Indian hospitals, antibiotic sensitivity tests have reported 30 to $80 \%$ of MRSA, which is similar to our study. Several authors have reported a substantial increase in MRSA prevalence in India. It has increased from 12\% in 1992 to $40 \%$ in 2009. Increasing resistance of MRSA in recent years has had a significant impact on several aspects of patient care and infection control. Methicillin resistance is often associated with simultaneous resistance for macrolides, quinolones and Cotrimoxazole [9].

The distribution of Enterococci among the various samples, where they were most commonly found in urine and least in blood was in concordance to the trends seen among other studies [10, 15]. The MRSA distribution was also similar to other reports from India, with maximum isolation among pus samples and least in blood and other body fluids [16], [17].

The drug resistance pattern in a study by Modi et al was similar to ours where he reported that Enterococci isolates were $100 \%$ sensitivite to Linezolid and $97.20 \%$ to Teicoplanin, however unlike our observation the isolates were $100 \%$ resistant to Penicillin and Ampicillin [4]. While studying the prevalence of multidrug-resistant Enterococci in a tertiary care hospital in Mumbai Despandya et al reported rate of resistance to drugs such as Ampicillin, Erythromycin, Ciprofloxacin, Vancomycin, Teicoplanin and Linezolid in percentages much higher than our study but on the other hand their Penicillin resistance was lower as compared to ours [18]. Penicillin resistance ranging from 1-29\% has been reported among Enterococcal isolates which is much lower than at our centre, whereas the resistance to Ampicillin was found to range from $0-67 \%$ which is comparable to our findings [14].

Recently Chitnis et al conducted a study in central India where, Staphylococcal strains showed comparatively lesser, and Enterococcus higher resistance than that observed by us. The same study and a few others showed lower resistance towards Clindamycin by MRSA as compared to our study $(62.5 \%)$.Clindamycin has been used successfully for the treatment of infection caused by MRSA in the past[12]. Kali et al conducted a study where MRSA isolates also showed higher resistance to Cotrimoxazole (82.3\%) and Ciprofloxacin (76.4\%) as compared to our data [9]. 
Like most of the recent studies our study also showed that none of the MRSA were resistant to Vancomycin by MIC testing using E test [9], [12] but five isolates demonstrated intermediate resistance to Vancomycin (VISA). Eight of the MRSA isolates had Vancomycin MIC $3 \mu \mathrm{g} / \mathrm{ml}$ and 24 of the MRSA had MIC between 1.5-2 $\mu \mathrm{g} / \mathrm{ml}$. Vancomycin has been the most reliable therapeutic agent against MRSA for the past three decades. However, despite its sustained in vitro microbiologic inhibitory activity, clinicians continue to debate its utility for MRSA infections. Significant controversy still exists regarding the current and future roles of Vancomycin in the treatment of serious MRSA infections. Vancomycin treatment failure is not uncommon, even when the MRSA strains are fully susceptible [breakpoint Minimum Inhibitory Concentration (MIC), $\leq 2 \mu \mathrm{g} / \mathrm{ml}$ ] to Vancomycin according to the Clinical and Laboratory Standards Institute (CLSI) criterion. Among MRSA strains for which Vancomycin MICs are elevated (1-2 $\mu \mathrm{g} / \mathrm{ml}$ ), failure of Vancomycin therapy or reduction in efficacy has been widely reported. These reports suggest that modest elevations in MICs may explain some suboptimal clinical outcomes. In addition, there is increasing number of reports showing discrepancies between in vitro susceptibility test results for Vancomycin and clinical outcomes of MRSA infections treated with Vancomycin [19]. While studying the in vitro activity of Daptomycin \& Linezolid against Methicillin resistant Staphylococcus aureus \& Vancomycin resistant Enterococci, Chitis et al in their study also showed a rise in MIC to Vancomycin for sizable number of MRSA and growing percentage of VRE like at our centre [12].

MRSA strains could develop resistance to Vancomycin through the acquisition of the Van A genes from VRE. In June 2002 the first clinical case of Van A mediated Vancomycin-resistant Staphylococcus aureus (VRSA) infection in the world was reported by the Michigan Department of Community Health. After that, 6 additional cases have been confirmed by the Centre for Disease Control and Prevention. The first VRSA clinical strain was confirmed to have a Van A gene sequence that was the same sequence as the Van A gene isolated from an Enterococcus faecalis obtained from an infected foot ulcer of the same patient. This is the first demonstration of the transfer of the Van A gene in vivo from E. faecalis to $S$. aureus; although such a transfer was achieved in 1992 in vitro. According to the clinical data of patients with confirmed VRSA in the US, several predisposing characteristics were found, including previous exposure with Vancomycin, severe underlying illness, and a history of MRSA and VRE infection or colonization. This suggests that, once MRSA strains and VRE strains occur in same patient or the surrounding environment at the same time, the probability of VRSA infections may increase [20].

By the E test method,in our study MRSA isolates showed no Teicoplanin resistance, although $4.55 \%$ showed intermediate resistance to the drug. Enterococcal isolates on the other hand showed $9.09 \%$ resistance to Teicoplanin. The MRSA and VRE strains demonstrated $97.73 \%$ susceptibility to Daptomycin, which was lower than that seen in other studies [9], [12]. Another group has reported $>99$ per cent susceptibility of Daptomycin against Staphylococci from Asia pacific region [21]. India Daptomycin Study Group reported Daptomycin MIC in the of range $0.047-1 \mu \mathrm{g} / \mathrm{ml}$ [22]. MIC for Daptomycin was found to be in the range of $0.064-1 \mu \mathrm{g} / \mathrm{ml}$ for 86 of the MRSA samples. Reduced susceptibility to Vancomycin has been reported to be associated with reduced susceptibility to Daptomycin as well. Diederen et al reported 7 of the 17 VISA isolates to have Daptomycin MIC of $2 \mu \mathrm{g} / \mathrm{ml}$ and one isolate to have MIC 4 $\mu \mathrm{g} / \mathrm{ml}$ [23]. Others have also shared similar experiences [21]. Sader et al showed bactericidal activity of Daptomycin against heterogenous VISA (hiVISA) isolated from blood [24]. They observed occurrence of Vancomycin tolerant and heterogenous Vancomycin intermediate strains (hiVISA) among S. aureus causing blood stream infection in nine USA hospitals. The use of Vancomycin to treat infections caused by MRSA having Vancomycin MIC $2 \mu \mathrm{g} / \mathrm{ml}$ also needs caution since therapeutic efficacy of Vancomycin in such situation may not be rewarding [12].

Linezolid has a broad spectrum activity against Gram-positive bacteria including multiple drug resistant isolates. It is active against both MSSA and MRSA, and inhibits all strains at a concentration of $4 \mu \mathrm{g} / \mathrm{ml}$ or less. Currently, Linezolid is the only oral agent approved by the Food and Drug Administration for treatment of infections caused by VRE. Existing mechanisms of resistance among other agents do not confer cross-resistance to Linezolid. Linezolid is the antiVRE drug used most commonly. [4]. A number of studies have also shown Linezolid to have excellent activity against MRSA and Enterococci like our centre [9], [12].

The disc diffusion method is not recommended for Vancomycin resistance detection, our study also showed much higher rate of resistance by this method compared to MIC determination by E test. The emergence of Vancomycinintermediate S.aureus strains and that of Vancomycin-resistant S.aureus strains are reasons for concern; these organisms still are extremely rare. Nevertheless, a number of studies have demonstrated increased clinical failure with MRSA isolates for which Vancomycin MICs are increased $(>1 \mu \mathrm{g} / \mathrm{ml})$ but still within the susceptibility range $(\leq 2$ $\mu \mathrm{g} / \mathrm{ml}$ ). Our findings suggest the requirement to re-look into the breakpoints for Vancomycin for determining sensitivity of MRSA isolates. Guidelines should also specify the method to be used for determining the MIC. Automated systems, which used to perform susceptibility testing, do not provide a precise Vancomycin MIC. The E test method is an alternative and feasible option for Vancomycin testing since it is easy to perform and cost-effective for testing only one drug for one strain. Interpretation of results is also easy. We recommend using E test as a routine test for determining MIC because of the above mentioned reasons. Agar dilution test should be done on all those strains showing higher MIC (intermediate or resistant results) by E test. However, routine use of agar dilution is cumbersome and labour intensive. If differences of only one dilution are relevant to predicting clinical outcomes of MRSA infections, the MIC method used is a critical part of the equation. Furthermore, the fact that the higher Vancomycin MIC results provided by the E test appear to be more reliable in predicting Vancomycin treatment responses, which indicates that the Vancomycin susceptibility breakpoint should be revaluated [19]. 


\section{Conclusion}

To conclude, elevated MIC for Vancomycin among MRSA and Enterococci is a cause of concern and Daptomycin, Teicoplanin and Linezolid remain good therapeutic alternatives to treat infections caused by MRSA and Enterococcus. Also, strict infection control measures need to be emphasized to control their prevalence in the hospital. The selection of antibiotic should be based on in vitro susceptibility, the hospital based antibiotic policies must be strictly followed and there should be constant surveillance of drug resistance for all bacterial pathogens [12].

Daptomycin was found to be highly active against all the MRSA and Enterococcal isolates tested, suggesting that Daptomycin testing should be included routinely. It is known to have rapid concentration-dependent bactericidal activity against $S$. aureus (both MSSA and MRSA) and Enterococcus faecalis (both Vancomycin susceptible and resistant) [25]. Considering its safety profile and rapid bactericidal action, and also ease of administration with once daily dose, Daptomycin may be useful for the treatment of severe infection caused by MRSA. It could be an alternative to Vancomycin in the treatment of infections where it is not possible to use Vancomycin due to resistance or nephrotoxicity. [26].

Combination therapy with a cell wall active agent and an aminoglycoside is recommended for serious Enterococcal infections but increasing resistance to first line antimicrobial agents and emergence and spread of species resistant to Vancomycin and high level Gentamicin have reduced therapeutic options to treat several Enterococcal infections [14]. Guidelines have been established to prevent the spread of resistant organisms. Each hospital needs to be familiar with the guidelines and establish a policy that reflects their unique needs.

The principle recommendations advocated are: 1) The prudent use of Vancomycin and other higher level drugs; 2) An ongoing education program for all hospital staff about the threat of resistant organisms; 3) A cooperative effort between clinicians and laboratory personnel that will allow the resistant organisms to be promptly and accurately detected; 4) The implementation of appropriate infection-control measures to prevent person-to-person spread of infection ; 5) Screening of health care workers in order to identify carrier rates 6) Surveillance cultures in high prevalence areas such as intensive care units and operation theatres 7) Knowledge of the antibiogram.

The accretion and spread of antibiotic resistance determinants among organisms, to the point where some clinical isolates are resistant to all standard therapies highlight both the vulnerability of our present armament as well as the looming prospect of a 'post antibiotic era'. Antibiotic policies need to be updated regularly, along with comprehensive monitoring of antibiotic prescribing and antibiotic consumption in healthcare settings.

\section{Acknowledgements}

We offer our sincere thanks to Novartis India Ltd for supplying the E test strips.

\section{References}

[1] Davies, Julian, and Dorothy Davies. Origins and evolution of antibiotic resistance. Microbiology and Molecular Biology Reviews, 74.3 (2010): 417-433.

[2] In Forbes BA, Sahm DF, Weissfeld AS, editors. Laboratory methods for detection of antibacterial resistance, Chapter 18. In Bailey and Scott's Diagnostic Microbiology. 10th ed. London: Mosby; 1998. p. 250-72. Back to cited text no. 6

[3] Woodford, Neil, et al. Current perspectives on glycopeptide resistance. Clinical Microbiology Reviews 8.4 (1995): 585-615.

[4] Modi, G. B., Soni, S. T., Patel, K. J.,et al. Prevalenace of Vancomycin Resistant Enterococci in Tertiary Care Hospital, Western, India. International Journal of Microbiology Research, Volume 4, Issue 2, 2012, pp.-182-185.

[5] Baird D. Staphylococcus: cluster-forming Gram positive cocci. 16. In: Collee AG, Fraser BP, Marmion JG, Simmons A, editors. Mackie and McCartney practical medical microbiology, 14th Ed. New York: Churchill Livingstone; 1996. p. 245-61.

[6] Facklam RR, Collins MD. Identification of 17. Enterococcus species isolated from human infections by a conventional test scheme. J Clin Microbiol 1989; 27: 731-4.

[7] Clinical Laboratory Standards Institution: Performance standards for antimicrobial susceptibility testing. In NCCLS approved standard M2A8. Wayne, PA USA: CLSI; 2004.

[8] Clinical and Laboratory Standards Institute. Performance standards for Antimicrobial Susceptibility Testing; Twenty-Third Informational Supplement. CLSI document M100-S23. Wayne, PA: Clinical and Laboratory Standards Institute, 2013:74.

[9] Kali, Arunava, et al. Changing Trends in Resistance Pattern of Methicillin Resistant Staphylococcus aureus. Journal of clinical and diagnostic research: JCDR 7.9, (2013): 1979.

[10] Desai PJ, Pandit D, Mathur M, Gogate A. The prevalence identification and the distribution of various species of enterococci which were isolated from clinical samples, with special reference to the urinary tract infections in catheterized patients. India J Med Microbial 2001; 19:132-7.

[11] Schaberg DR, Culver DH, Gaynes RP. Major trends in the microbial etiology of nosocomial infection. Am J Med 1991; 91: 72S-75S

[12] Chitnis, Sheetal, et al. In vitro activity of daptomycin \& linezolid against methicillin resistant Staphylococcus aureus \& Vancomycin resistant enterococci isolated from hospitalized cases in Central India.The Indian journal of medical research 137.1 (2013): 191.

[13] Telkar, A. Change in the Prevalence and the Antibiotic Resistance of the Enterococcal Species Isolated from Blood Cultures.

[14] Pandya, A., Shah, M. B., Trivedi, S., \& Singh, S. Identification, characterization and antimicrobial susceptibility testing of Enterococcus species isolated from various clinical specimens.

[15] Sharma, R., \& Pai, C. Prevalence of Various Enterococcal Infections and Its Antibiotic Susceptibility with Special Reference to Vancomycin and High Level Gentamicin Resistance in a Tertiary Care Centre in Navi Mumbai.

[16] Ranjan KP, Arora DR, Ranjan N. An approach to Linezolid and Vancomycin against Methicillin resistant Staphylococcus Aureus. Webmed Central MICROBIOLOGY 2010; 1:WMCOO590. 
[17] Rajaduraipandi, K., et al. Prevalence and antimicrobial susceptibility pattern of methicillin resistant Staphylococcus aureus: A multicentre study. Indian Journal of Medical Microbiology. 24.1 (2006): 34.

[18] Deshpande, Vaibhav R., Mohan G. Karmarkar, and Preeti R. Mehta. Prevalence of multidrug-resistant enterococci in a tertiary care hospital in Mumbai, India. The Journal of Infection in Developing Countries. 7.02 (2013): 155-158.

[19] Tandel, K., A. K. Praharaj, and S. Kumar. Differences in Vancomycin MIC among MRSA isolates by agar dilution and E test method. Indian journal of medical microbiology. 30.4. (2012): 453.

[20] Wang, Zhen, et al. Investigation of the prevalence of patients co-colonized or infected with methicillin-resistant Staphylococcus aureus and Vancomycin-resistant enterococci in China: a hospital-based study. Chin Med J. 122.11 (2009): 1283-1288.

[21] Biedenach DJ, Bell JM, Sader HS, Fritsche TR, Jones RN, 6. Turnidge JD. Antimicrobial susceptibility of gram positive bacterial isolates from the Asia Pacific region and an in vitro evaluation of the bactericidal activity of daptomycin, Vancomycin and teicoplanin: a SENTRY program report (2003-2004). Int J Antimicrob Agents. 2007; 30: 143-9.

[22] Mathai D, Biedenbach DJ, Jones RN, Bell JM, Turnidge 14. J Sader HS; India Daptomycin Study group. Activity of daptomycin against Gram positive bacterial isolates from Indian medical centre (2006-2007). Int J Antimicrob Agents. 2009; 34: 497-9.

[23] Diederen BMW, Duijn IV, Willemse P, Klutymans JW. 5. In vitro activity of daptomycin against methicillin resistant Staphylococcus aureus including heterogeneously glycopeptide resistant strains. Antimicrob Agents Chemother. 2006; 50: 3189-91.

[24] Sader HS, Wallers AA, Fritsche TR, Jines RN. Daptomycin 8. Antimicrobial activity tested against methicillin resistant staphylococci and Vancomycin resistant enterococci isolated in European medical centres (2005). BMC Infect Dis. 2007; 18: 29.

[25] Philip IH, Susan JK. Datpomycin a review of its use in the 3. Management of complicated skin and soft tissue infections and Staphylococcus aureus bacteraemia. Drug .2007; 67: 1483-512.

[26] Kaur, Rajneet, et al. Daptomycin susceptibility of methicillin resistant Staphylococcus aureus (MRSA). The Indian journal of medical research.136.4 (2012): 676. 